\title{
Mitteilungen der Westdeutschen Gesellschaft für Pneumologie
}

Pneumologe 2016 $\cdot 13: 360$

DOI 10.1007/s10405-016-0069-7

c) Springer-Verlag Berlin Heidelberg 2016

Vorsitzende

Prof. Dr. med. J. Lorenz

Klinikum Lüdenscheid -

Klinik für Pneumologie

und Internistische Intensivmedizin

Paulmannshöher Str. 14, 58515

Lüdenscheid
Kongresspräsident

Dr. med. P. Haidl

Chefarzt Pneumologie II

Ärztlicher Direktor,

Fachkrankenhaus Kloster Grafschaft Annostr. 1, 57392 Schmallenberg

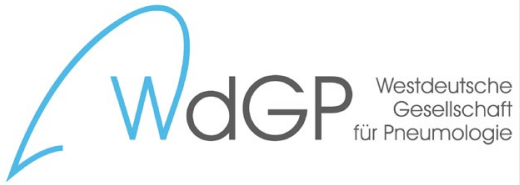

Geschäftsführer

Dr. med. T. Voshaar

Med . Klinik III

Schwerpunkt Pneumologie, Allergo-

logie, Schlaf- und Beatmungsmedizin:

Interdisziplinäres Lungenzentrum

Krankenhaus Bethanien

Bethanienstr. 21, 47441 Moers

\section{Arbeitsanleitungen zur Qualitätssicherung in Diagnostik und Therapie interstitieller Lungenerkrankungen}

Unsere Gesellschaft setzt einen Schwerpunkt in der Förderung der Qualitätssicherung auf dem Gebiet interstitieller und anderer seltener Lungenerkrankungen.

Aktuelle Fortschritte in der Erforschung dieser komplexen Erkrankungen ermöglichen heute eine präzise Diagnose. Sie ist die Voraussetzung für evidenzbasierte spezifische Therapien. Die Identifizierung und die Therapie pulmonaler Verlaufsformen von Systemerkrankungen wie Kollagenosen und Vaskulitiden oder die Behandlung spezieller Verlaufsformen, zum Beispiel die der steroidrefraktären Sarkoidose, haben jetzt eine robuste empirische Basis. Erstmals kann seit wenigen Jahren der Verlauf der idiopathischen Lungenfibrose medikamentös günstig beeinflusst werden. Wir Pneumologen können diese Erkenntnisse für unsere Patienten nur nutzen, wenn wir sowohl im ambulanten wie im stationären Bereich auf der Höhe der Zeit sind.

Die Bildung multidisziplinärer Teams mit Pathologen, Radiologen und Spezialisten für extrapulmonale Manifestationen ist eine wesentliche Voraussetzung für die Transmission der medizinischen Fortschritte in den Alltag. Die Pneumologie muss diese Herausforderung annehmen und in der Bildung solcher klinischer Arbeitsgruppen vorangehen.

Die Arbeitsgruppe interstitielle Lungenerkrankungen (AGILD) in der WDGP hat für einige wesentliche praktische Schritte im Versorgungsalltag Arbeitsanleitungen entworfen, die zu einer Vereinheitlichung und Standardisierung in der Behandlung beitragen sollen. Weitere werden folgen. Die hier vorgestellten Dokumente wurden jetzt von der WDGP freigegeben. Weitere Dokumente werden folgen. Sie sollen zur Diskussion über die Qualitätssicherung in der Pneumologie beitragen und können gerne von den Lesern weiterverwendet werden. Für Anmerkungen, Kritik und für Nachahmung/Erweiterung sind wir dankbar.

Frank Feldmeyer, Hagen und Joachim Lorenz Lüdenscheid für die AG-ILD

\section{Zusatzmaterial online}

In der Online-Version dieses Artikels (doi:10.1007/s10405-016-0069-7) finden Sie die Dokumente zu:

1. Laufzettel zur diagnostischen Abklärung von Patienten mit ILD

2. Anamnesebogen für interstitielle/diffuse Lungenerkrankungen

3. Screeninglabor bei interstitiellen Lungenerkrankungen und Immundiagnostik bei Verdacht auf exogen-allergische Alveolitis

4. Die bronchoalveoläre Lavage in der Diagnostik von ILD

5. Empfehlungen zur Computertomographie in der Diagnostik von ILD

6. Optionen in der Therapie von ILD 